\title{
A load off the mind
}

AutOMATION in the laboratory is like automation anywhere, but more so. Immediately after the Second World War, it seemed in all kinds of laboratories to be sensible that the repetitive measurement of the same properties of similar samples should be made by using the techniques developed by mechanical engineers for assembling bits and pieces of motor cars into standard structures - plastic bottles containing predetermined amounts of solutions doped with known radioactive isotopes would in this spirit be carried within the range of suitable detectors, scintillation counters perhaps. Other repetitive laboratory jobs the chemical analysis for specified elements of broadly similar specimens of material, for example - were made more or less automatic once there had been developed reliable devices for measuring small amounts of chemical reagents (usually in liquid form). Economically, such devices were easily justifiable; better that routine measurements and assays should be carried out by standardized procedures undisturbed by the personal equations of the experimenters, while the cost of employing laboratory assistants to carry out tasks that could be delegated to machines was often higher than that of the interest paid on large sums of money borrowed from some bank. Production-line automation in this style remains as productive of improved efficiency as at any time in the past three decades. Automatic amino acid analysers, or machines that will assemble some predetermined nucleotide sequence from its component nucleotides, have become essential components of many laboratories' equipment.

The notes in the pages that follow show, however, that the ambitions of those who would make laboratories automatic have now transcended those fashionable in earlier decades. At the bench, or at the control panel of some large telescope, people are now rightly concerned to ask whether the arrival of microprocessors provides an opportunity for delegating to machines tasks that can in principle be carried out only by people, but which are in practice likely to be performed only hesitantly, even inefficiently. Of all automatic laboratory instruments, the machine-driven telescope (dating from the early nineteenth century) is the prototype: even clockwork machinery is usually more dependable than a human being at following the rotation of the Earth on its axis.

Traditionally, astronomers have supposed that only human beings (people like themselves) can decide for how long a telescope should be locked onto some celestial object of particular interest before the equipment they are using can be pointed at something else. (Given the logarithmic response of the photographic emulsion to integrated light intensity, mistakes have traditionally been commonplace.) Fortunately, the high cost and, more to the point, the competition for access to machines such as the Einstein X-ray observatory have reminded astronomers of the need to work out in advance of the collection of radiation from a nominated celestial object the amount of radiation (the number of quanta) that will yield a significant result. One obvious snag, of course, is that the significance of a data-set thus collected automatically may depend critically on the quality of the data, so that it will not be possible to specify in advance how many quanta should be collected but only to work out as the data accumulate when an observation should be stopped. That is a problem that those who design experiments in high-energy physics have lived with for several years. What they have found is that the automated laboratory brings severe and unusual disciplines. The hypothesis to be tested must be clearly specified, and there must be explicit criteria for deciding what constitutes a significant test. Not unnaturally, in fields where the choice between manual and automatic experimentation is not overwhelmingly determined by the decrease of cost that automation brings, people resent the need to be this clear in advance.

These uncharted fields happen also to be those in which there is at present most interest, among manufacturers and their customers, in the automation of laboratory equipment. Two important influences have helped to bring about this state of affairs. First, the proportions of working scientists now reasonably skilled at writing computer programs has surreptitiously increased so that the notion that an experimental program might with advantage be delegated to a machine is more likely to come up than not. Second, the microprocessor has become ubiquitous and at the same time cheap. It is therefore natural enough that there should be a rash of laboratories concerned with traditional fields of research, muscle physiology or old-fashioned biochemical investigations, in which ingenious people have found it worthwhile (or at least fun) to latch together a microprocessor (perhaps in the form of a "home" computer) and an experimental rig in such a way as to avoid the need for telling when one "run" is at an end, and that it is time for another to begin. It is more of a surprise that equipment manufacturers rightly alert to the opportunities for adding to their catalogues equipment incorporating microprocessors should so often have found it necessary or at least expedient to quote substantially increased prices for automatic equipment compared with the traditional variety. Although users appreciate the need to amortize the cost of new developments, and even appreciate that their own increased efficiency is worth paying for, there is also a danger that manufacturers will rob themselves of valuable opportunities, and deprive their customers of important benefits, if they persist in seeking for themselves the whole of the value that can be added by automation.

In reality, the developments of the past few decades in the automation of experimental work are probably no more than a modest and even grudging concession to the practical importance of computing machinery. So much, it is hoped, will be apparent from Professor Donald Michie's article on computer chess (see page 391). This game is properly regarded as a kind of simulation of the problem of understanding how the natural world is put together. There is a qualitative correspondence between the search for winning strategies and that for successful hypotheses about the origins of natural phenomena. Computationally, chess is for the time being beyond the reach of even very large computers, implying that the understanding of natural phenomena is even less likely to be turned over to machines. But the past decade has seen such remarkable improvements in the capacity of machines to play the mechanical game of chess respectably, and the way ahead so clearly points to the utility of abstract definitions of useful strategies, that it is not unreasonable to hope that the practice of science may in due course be rid of the tedium by which generations of students (and even their teachers) have been oppressed. The drift towards the automation of the laboratory may be sustained by economic calculations turning on the salaries of technicians, but the long-term objective must be to free the imagination, not to cramp it. 